DOI: 10.12731/2658-6649-2020-12-1-92-97

УДК 51-7

\title{
QSPR МОДЕЛИРОВАНИЕ ТЕПЛОЕМКОСТИ АЛЬДЕГИДОВ
}

\section{Осипов А.Л., Трушина В.П., Осипов Ф.Л.}

B статье исследуются QSPR модели предсказания теплоемкости химических веществ семейства альдегидов. Исследование параметра теплоемкости осуществляется с помощью разработанных моделей с использованием следующих факторов: топологических индексов; структурных дескрипторов; информационного индекса, связанного с функиией Шеннона. Проведены вычислительные эксперименты, показывающие высокую эффективность предложенных QSPR зависимостей.

Ключевые слова: предсказание; брутто-формула; топологические индексы; структурные дескрипторы; информационный индекс; альдегиды; регрессионный анализ; теплоемкость.

\section{QSPR SIMULATION OF HEAT CAPACITY OF ALDEHYDES}

\author{
Osipov A.L., Trushina V.P., Osipov F.L.
}

The article explores QSPR models for predicting the heat capacity of chemicals in the aldehyde family. The study of the heat capacity parameter is carried out using the developed models using the following factors: topological indices; structural descriptors; information index associated with the Shannon function. Computational experiments were performed showing the high efficiency of the proposed QSPR dependencies.

Ключевые слова: prediction; gross formula; topological indices; structural descriptors; information index; aldehydes; regression analysis; heat capacity.

\section{Введение}

Одной из наиболее актуальных задач в химических исследованиях является проблема моделирования параметра теплоемкости с помощью QSPR моделей. Информация о теплоемкости химических веществ нуж- 
на для: вычисления тепловых балансов; проектирования теплообменной аппаратуры; расчета химического равновесия; определения энтропии вещества. Изучение механизмов теплоемкости химического вещества несет важную информацию о строении его молекул.

Незнание параметра теплоемкости существенно ограничивает практическую применимость химических веществ. В связи с этим в анализе QSPR зависимостей лучше разрабатывать статистические модели и информационные системы с базами химических соединений, обладающих параметром теплоемкости для определенных классов химических веществ.

Актуальность статьи заключается в конструировании новых QSPR моделей для предсказания параметра теплоемкости с учетом топологических и структурных дескрипторов, а также информационного индекса. В работе для моделирования параметра теплоемкости $C_{V}$ предлагаются дескрипторы, вычисление которых требуют знаний только структурной формулы химических соединений класса альдегидов.

\section{Методы исследования}

В качестве научных исследований используются методы: хемоинформатики, количественной связи структура - свойство; программирования; теории графов; моделирования и прогнозирования.

\section{Результаты исследования}

В качестве экспериментальной выборки были взяты 24 химических вещества из семейства альдегидов [1, с. 241] с рассчитанными топологическими индексами.

В таблице 1 представлены альдегиды с рассчитанными топологическими индексами, структурными дескрипторами и информационным индексом. Индекс Рандича обозначается $\chi$, Валабана - J, Харари - $H$, Винера $-W$, Шеннона $-I$.

Таблицуа 1.

Выборка альдегидов

\begin{tabular}{|c|c|c|c|c|c|c|}
\hline $\begin{array}{c}\text { Брутто- } \\
\text { формула }\end{array}$ & $\chi$ & $J$ & $H$ & $W$ & $I$ & $\begin{array}{c}\text { Количество } \\
C_{2}\end{array}$ \\
\hline $\mathrm{C}_{2} \mathrm{H}_{4} \mathrm{O}$ & 1,41 & 1,63 & 2,5 & 4 & 1,477733 & 0 \\
\hline $\mathrm{C}_{3} \mathrm{H}_{6} \mathrm{O}$ & 1,91 & 1,97 & 4,33 & 10 & 1,394096 & 1 \\
\hline $\mathrm{C}_{4} \mathrm{H}_{8} \mathrm{O}$ & 2,41 & 2,19 & 6,42 & 20 & 1,329252 & 2 \\
\hline $\mathrm{C}_{5} \mathrm{H}_{10} \mathrm{O}$ & 2,91 & 2,34 & 8,7 & 35 & 1,280385 & 3 \\
\hline $\mathrm{C}_{6} \mathrm{H}_{12} \mathrm{O}$ & 3,41 & 2,45 & 11,15 & 56 & 1,242608 & 4 \\
\hline
\end{tabular}


Окончание табл. 1.

\begin{tabular}{|c|c|c|c|c|c|c|}
\hline $\mathrm{C}_{7} \mathrm{H}_{14} \mathrm{O}$ & 3,91 & 2,53 & 13,74 & 84 & 1,212573 & 5 \\
\hline $\mathrm{C}_{8} \mathrm{H}_{16} \mathrm{O}$ & 4,41 & 2,6 & 16,46 & 120 & 1,188102 & 6 \\
\hline $\mathrm{C}_{9} \mathrm{H}_{18} \mathrm{O}$ & 4,91 & 2,65 & 19,26 & 165 & 1,167756 & 7 \\
\hline $\mathrm{C}_{10} \mathrm{H}_{20} \mathrm{O}$ & 5,41 & 2,69 & 22,22 & 220 & 1,15055 & 8 \\
\hline $\mathrm{C}_{11} \mathrm{H}_{22} \mathrm{O}$ & 5,91 & 2,73 & 25,24 & 286 & 1,135791 & 9 \\
\hline $\mathrm{C}_{12} \mathrm{H}_{24} \mathrm{O}$ & 6,41 & 2,76 & 28,34 & 364 & 1,122977 & 10 \\
\hline $\mathrm{C}_{13} \mathrm{H}_{26} \mathrm{O}$ & 6,91 & 2,78 & 31,52 & 455 & 1,111737 & 11 \\
\hline $\mathrm{C}_{14} \mathrm{H}_{28} \mathrm{O}$ & 7,41 & 2,81 & 34,77 & 560 & 1,101789 & 12 \\
\hline $\mathrm{C}_{15} \mathrm{H}_{30} \mathrm{O}$ & 7,91 & 2,83 & 38,09 & 680 & 1,092915 & 13 \\
\hline $\mathrm{C}_{16} \mathrm{H}_{32} \mathrm{O}$ & 8,41 & 2,85 & 41,47 & 816 & 1,084945 & 14 \\
\hline $\mathrm{C}_{17} \mathrm{H}_{34} \mathrm{O}$ & 8,91 & 2,86 & 44,91 & 969 & 1,077743 & 15 \\
\hline $\mathrm{C}_{18} \mathrm{H}_{36} \mathrm{O}$ & 9,41 & 2,88 & 48,41 & 1140 & 1,071199 & 16 \\
\hline $\mathrm{C}_{19} \mathrm{H}_{38} \mathrm{O}$ & 9,91 & 2,89 & 51,95 & 1330 & 1,065225 & 17 \\
\hline $\mathrm{C}_{20} \mathrm{H}_{40} \mathrm{O}$ & 10,41 & 2,9 & 55,55 & 1540 & 1,059746 & 18 \\
\hline $\mathrm{C}_{21} \mathrm{H}_{42} \mathrm{O}$ & 10,91 & 2,91 & 59,2 & 1771 & 1,054701 & 19 \\
\hline $\mathrm{C}_{22} \mathrm{H}_{44} \mathrm{O}$ & 11,41 & 2,92 & 62,89 & 2024 & 1,050039 & 20 \\
\hline $\mathrm{C}_{23} \mathrm{H}_{46} \mathrm{O}$ & 11,91 & 2,93 & 66,62 & 2300 & 1,045717 & 21 \\
\hline $\mathrm{C}_{24} \mathrm{H}_{48} \mathrm{O}$ & 12,41 & 2,94 & 70,4 & 2600 & 1,041697 & 22 \\
\hline $\mathrm{C}_{25} \mathrm{H}_{50} \mathrm{O}$ & 12,91 & 2,95 & 74,21 & 2925 & 1,037948 & 23 \\
\hline
\end{tabular}

В статье в качестве информационного индекса $I$ используется величина, вычисляемая по формуле $I=-\sum_{i=1}^{N} \frac{n_{i}}{N} \log \frac{n_{i}}{N}$, где $n_{i}$ - число атомов $i$-го сорта, а $N$ - общее число атомов в молекуле. Индекс $I$ программно вычислялся по брутто-формуле. В качестве структурного дескриптора использовался фактор, показывающий количество фрагментов $\mathrm{CH}_{2}$ в молекуле химического соединения.

В работе [1, c. 238] представлена QSPR модель предсказания параметра теплоемкости на основе топологических индексов $C_{V}=0,817-1,034 \mathrm{~J}+$ $+0,0001 W+9,182 \chi$. Параметры модели следующие: коэффициент детерминации $R^{2}=1$; стандартная ошибка $S D=0,267$; критерий Фишера $F=$ 108300 ; средняя абсолютная ошибка в \% $M A P E=0,3845$; средняя абсолютная ошибка $M A E=0,1435$.

B статье разработаны следующие QSPR модели предсказания параметра теплоемкости, представленные в таблице 2. 
Таблица 2.

Характеристики моделей для предсказания теплоемкости

\begin{tabular}{|l|c|c|c|c|c|}
\hline \multicolumn{1}{|c|}{ Модель } & $R^{2}$ & $F$ & $S D$ & MAPE & MAE \\
\hline$C_{V}=-2,431+9,193 \mathrm{I}+5,484 \mathrm{CH}_{2}-0,273 H$ & 1 & 115077,9 & 0,2586 & 0,3687 & 0,1367 \\
\hline$C_{V}=64,09-39,2974 I+1,2259 H$ & 1 & 18905,2 & 0,7813 & 1,7429 & 0,5816 \\
\hline$C_{V}=10,14+4,5031 \mathrm{CH}_{2}-0,0069 H$ & 1 & 140386,5 & 0,2868 & 0,4727 & 0,1344 \\
\hline $\begin{array}{l}C_{V}=0,0344 I+1,2238 \mathrm{CH}_{2}-0,1934 H \\
\text { для стандартизированных переменных }\end{array}$ & 1 & 120831,8 & 0,2361 & 0,0037 & 0,1367 \\
\hline$C_{V}=4,01 \cdot I^{0,1261} \cdot \mathrm{CH}_{2}^{-0,1194} \cdot H^{0,8609}$ & 1 & 29514,6 & 0,2438 & 0,4125 & 0,1519 \\
\hline
\end{tabular}

Из анализа приведенных моделей видно, что стандартная ошибка у трех приведенных выше моделей лучше, чем в работе [1, с. 238]. Более того эти модели более просты в вычислительном отношении. Расчет по моделям проводился с помощью MS Excel и статистического пакета SPSS [2, с. 57].

\section{Обсуждение}

Предлагаемая методология на основе количественной связи «структура молекул - свойства» позволяет достаточно эффективно предсказывать теплоемкость по достаточно просто вычисляемым факторам. Поэтому разработанные QSPR модели можно рекомендовать для практического использования.

\section{Заключение}

Предложены QSPR модели для исследования взаимосвязи параметра теплоемкости альдегидов от структурных, топологических и информационных факторов. Проведено сравнение разработанных моделей с уже существующими подходами. Среди предложенных моделей выбраны наилучшие.

Информация о конфликте интересов. Конфликт интересов отсутствует.

Информация о спонсорстве. Спонсорская поддержка отсутствует.

\section{Список литературы}

1. Alaghebandi A. QSPR modeling of heat capacity, thermal energy and entropy of aliphatic aldehydes by using topological indices and MLR method / Alaghe- 
bandi A., F. Shafiei // Iranian Journal of Mathematical Chemistry. Vol 7. No 2. 2016, pp. 235-251.

2. Бюль А., Цефель П. SPSS: искусство обработки информации. СПб: ДиаСофтЮП, 2005. 608 с.

\section{References}

1. Alaghebandi A., Shafiei F. QSPR modeling of heat capacity, thermal energy and entropy of aliphatic aldehydes by using topological indices and MLR method. Iranian Journal of Mathematical Chemistry. Vol 7. No 2. 2016, pp. 235-251.

2. Byul' A., Cefel' P. SPSS: iskusstvo obrabotki informacii [SPSS: the art of information processing]. SPb: DiaSoftYUP. 2005. 608 p.

\section{ДАННЫЕ ОБ АВТОРАХ}

Осипов Александр Леонидович, доцент, кандидат технических наук ФГБОУ ВО Новосибирский государственный университет экономики и управления

ул. Каменская, 56, г. Новосибирск, 630099, Российская Федерация alosip@mail.ru

Трушина Вероника Павловна, старший преподаватель ФГБОУ ВО Новосибирский государственный университет экономики и управления

ул. Каменская, 56, г. Новосибирск, 630099, Российская Федераиия veronika07-92@mail.ru

Осипов Федор Леонидович, доцент, кандидат педагогических наук ФГБОУ ВО Новосибирский государственный университет экономики и управления ул. Каменская, 56, г. Новосибирск, 630099, Российская Федераиия alosip@mail.ru

\section{DATA ABOUT THE AUTHORS}

Osipov Alexander Leonidovich, Associate Professor, Candidate of Technical sciences

Novosibirsk State University of Economics and Management 56, Kamenskaya str., Novosibirsk, 630099, Russian Federation alosip@mail.ru 
Trushina Veronica Pavlovna, Senior Teacher

Novosibirsk State University of Economics and Management 56, Kamenskaya str., Novosibirsk, 630099, Russian Federation veronika07-92@mail.ru

Osipov Fedor Leonidovich, Associate Professor, Candidate of Pedagogical Sciences

Novosibirsk State University of Economics and Management 56, Kamenskaya str., Novosibirsk, 630099, Russian Federation alosip@mail.ru 